Research Article

\title{
Clinical Value of Serum Thrombospondin-2 Combined with CA19-9 in Early Diagnosis of Gastric Cancer
}

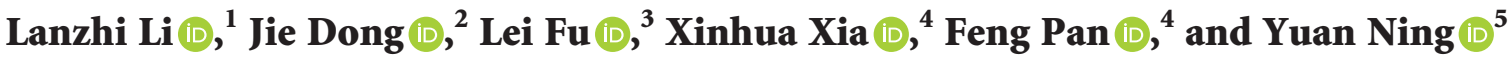 \\ ${ }^{1}$ Health Examination Department, Yantaishan Hospital, Yantai 64000, China \\ ${ }^{2}$ Department of Clinical Laboratory, Yantai Yuhuangding Hospital Affiliated To Qingdao University, Yantai 264000, China \\ ${ }^{3}$ PIVAS, Affiliated Qingdao Central Hospital, Qingdao University, Qingdao 266000, China \\ ${ }^{4}$ Ward Department, Zhangqiu District People's Hospital, Jinan 250200, China \\ ${ }^{5}$ Supply Support Center, The Central Hospital Affiliated to Shandong First Medical University, 105 Jiefang Road, \\ Jinan 250013, Shandong, China
}

Correspondence should be addressed to Yuan Ning; yuanning@jnzxhospital.cn

Received 1 June 2021; Revised 14 July 2021; Accepted 15 September 2021; Published 7 October 2021

Academic Editor: Alamgeer Yuchi

Copyright (c) 2021 Lanzhi Li et al. This is an open access article distributed under the Creative Commons Attribution License, which permits unrestricted use, distribution, and reproduction in any medium, provided the original work is properly cited.

Gastric cancer (GC) is a kind of common cancer worldwide. Too late in diagnosis results in poor prognosis of patients with GC. Thrombospondin-2 (THBS2) is a type of secreted protein that has been found to be a diagnostic biomarker in a variety of cancers. Our study aimed to uncover the clinical value of THBS2 in early detection for patients with gastric cancer. THBS2 was upregulated in gastric cancer tissue compared with normal tissue via analyzing data obtained from The Cancer Genome Atlas (TCGA) database. Additionally, the enzyme-linked immunosorbent assay revealed that the level of serum THBS2 and carcinoembryonic antigen, CA19-9, was higher dramatically in patients with early gastric cancer (EGC) than that in healthy control (HC) in addition to patients with benign gastric tumor (BGT), which suggested that THBS2 indeed associated with GC. Receiver operator characteristic (ROC) curve assay was conducted to demonstrate that serum THBS2 was similar to CA19-9 to distinguish patients with early gastric cancer from healthy control and patients with benign gastric tumor and that THBS2 combined with CA19-9 improved the detective performance of THBS2 for early gastric cancer. Furthermore, we applied the gene set enrichment analysis assay to analyze signaling pathways related to THBS2. We found that THBS2 positively controlled MAPK and WNT signaling pathways, which indicated that THBS2 might exert its functions via the pathway mentioned above. Thus, our study expounded that serum THBS2 could serve as a vital early diagnostic marker for patients with gastric cancer.

\section{Introduction}

Gastric cancer (GC) as the fourth leading tumor results in third most main death rate that is related to cancers in the world [1]. The disease incidence and mortality of GC maintains high in both East Asian and Central South American countries including China, Korea, Mexico, and Chile $[2,3]$. Early gastric cancer can invade either mucosa or submucosa, which is accompanied by metastasis of the lymph node or not [4]. Late detection, in addition to insensitivity to existing treatment options, causes a poor prognosis of GC [5]. For an optimum period of therapy, it is necessary to diagnose GC as early as possible. Recently, advanced technologies such as screening are employed to raise diagnosis proportion of early gastric cancer [6]. Carcinoembryonic antigen, CA19-9, and other detection methods are available to predict GC [7]. Serum markers are easier to detect than other test indexes; therefore, they are used widely to diagnose early gastric cancer [8]. The most sensitive serum index for diagnosing GC, however, is still uncovered [9]. Thus, it is urgent to explore new and highly effective biomarkers to boost the detective accuracy of early GC [10].

Thrombospondins (THBSs), a family belonging to $\mathrm{Ca}^{2+}$ binding glycoproteins, are secretions of immune cells and mesenchymal cells in addition to endotheliocytes [11]. They 
can bind to plenty of downstream proteins involved in various biological procedures, including cell migration, blood vessel production, apoptosis, and cytoskeletal regulation $[12,13]$. In multiple malignancies, THBS2 is tight correlated with progression and prognosis of cancers [14]. Tokunaga et al. discovered that the expression of THBS2 in colon cancer decreased hepatic metastases risk as well as angiogenesis of tumors compared to patients lacking THBS2 [15]. Additionally, THBS2 had been found to exert a vital impact on lung adenocarcinoma, prostate cancer, myeloid tumor, and breast cancer [16, 17]. Nevertheless, the specific effect of THBS2, in addition to its clinical value for gastric cancer, is not disclosed now. Therefore, we are trying to expose its clinical significance in the detection of early gastric cancer.

\section{Materials and Methods}

2.1. Patients and Samples. This is a prospective study. Blood was obtained from 41 healthy individuals, 33 benign gastric tumor patients, and 46 early gastric cancer patients at Yantaishan Hospital, Yantai, China. The benign or early stage of cancer was confirmed by the principle of the American Joint Committee on Cancer (AJCC) TNM (tumor-node-metastasis) classification. Blood was centrifuged; then, serum was collected and stored at $-80^{\circ} \mathrm{C}$ freezer. Basic information such as age, gender, and amounts of samples are given in Table 1. All participants voluntarily participated in our studies, and all protocols related to human volunteers were abiding by the guidance of the Helsinki Declaration. All patients provided written informed consent, and the study was approved by the Ethics Committee of the Yantaishan Hospital, Yantai, China.

2.2. Gene Set Enrichment Analysis (GSEA). To verify the molecular signaling pathway related to the high expression of THBS2, GSEA was conducted by using GSEA 3.0 software. Specific gene sets were downloaded from the official website (http://software.broadinstitute.org/gsea/index.jsp) and analyzed for pathway enrichment.

2.3. Enzyme-Linked Immunosorbent Assay (ELISA). The protein levels of THBS2 and CA19-9 in serum were examined by the ELISA kit (ThermoFisher, USA). Serum from early gastric cancer (EGC), benign gastric tumor (BGT), or healthy control (HC) was incubated with a microtiter plate which had been treated with capture antibodies for 1 hour at room temperature. Then, the plate was washed and incubated with a specific antibody for 0.5 hour at room temperature, followed by washing the plate. Then, the secondary antibody was added into plate, and reaction was terminated by a stop solution. A microplate reader (BioRad, CA) was employed to record the absorbance at $450 \mathrm{~nm}$. The detailed protocol was in accordance with the reagent specification.

2.4. Statistical Analysis. All data were analyzed by using SPSS 19.0 software (IBM, USA) and GraphPad 8.0 software (CABIT, China) for calculating statistical significance and
TABLE 1: Basic information.

\begin{tabular}{lccc}
\hline Diagnosis & Healthy control & $\begin{array}{c}\text { Benign gastric } \\
\text { tumor }\end{array}$ & $\begin{array}{c}\text { Early gastric } \\
\text { cancer }\end{array}$ \\
\hline Sex & 22 & 15 & \\
Male & 19 & 18 & 25 \\
Female & & & 21 \\
\hline Age & 55 & 53 & 58 \\
Mean & $21-67$ & $20-65$ & $24-71$ \\
Range & 41 & 33 & 46 \\
Amount & & & \\
\hline
\end{tabular}

TABLE 2: Data of ROC curve.

\begin{tabular}{lcc}
\hline & AUC & $95 \%$ CI \\
\hline HC vs. EGC & & \\
THBS2 & 0.816 & $0.722-0.911$ \\
CA19.9 & 0.901 & $0.833-0.968$ \\
THBS2 + CA19.9 & 0.951 & $0.912-0.989$ \\
\hline BGT vs. EGC & & \\
THBS2 & 0.840 & $0.752-0.927$ \\
CA19.9 & 0.847 & $0.762-0.931$ \\
THBS2 + CA19.9 & 0.928 & $0.872-0.984$ \\
\hline
\end{tabular}

${ }^{*} P<0.05$ in comparison with CA19.9.

was presented in a manner of mean \pm standard deviation (SD). The correlation between THBS2 and CA19-9 was measured by Pearson's correlation analysis. Receiver operator characteristic (ROC) curves were drawn to estimate the diagnostic performance. All relevant data are given in $\mathrm{Ta}$ ble $2 . P<0.05$ is statistically significant.

\section{Results}

3.1. The Level of THBS2 Is Increased in Gastric Cancer Tissues. The load of THBS2 in tumor tissues and normal tissues of patients diagnosed with gastric cancers was analyzed by deferential analysis as well as paired differential analysis on the basis of data from The Cancer Genome Atlas (TCGA) database. The mean level of THBS2 in gastric cancer tissues was obviously higher than that in control tissues $(P<0.001$, Figures $1(\mathrm{a})$ and $1(\mathrm{~b})$ ). The data showed that serum THBS2 was potential to be secreted by gastric cancer tissues.

3.2. Increased Level of THBS2 in Serum of Patients with Early Gastric Cancer. To confirm results mentioned above, an ELISA was conducted to measure the load of serum THBS2 in 46 patients with EGC or 33 patients with BGT in addition to $41 \mathrm{HC}$. The results illustrated that the protein level of serum THBS2 in people with EGC was upregulated dramatically compared with that in $\mathrm{HC}$ and BGT patients. Additionally, the load of serum THBS2 in BGT patients was higher than that in $\mathrm{HC}(P<0.05$, Figure $2(\mathrm{a}))$. CA19-9, a common biomarker for GC, was also estimated by the ELISA assay. As shown in Figure 2(b), the level of serum CA19-9 was the highest in EGC patients while the lowest in $\mathrm{HC}$, which is consistent with the load of serum THBS2 $(P<0.05$, Figure 2(b)). 


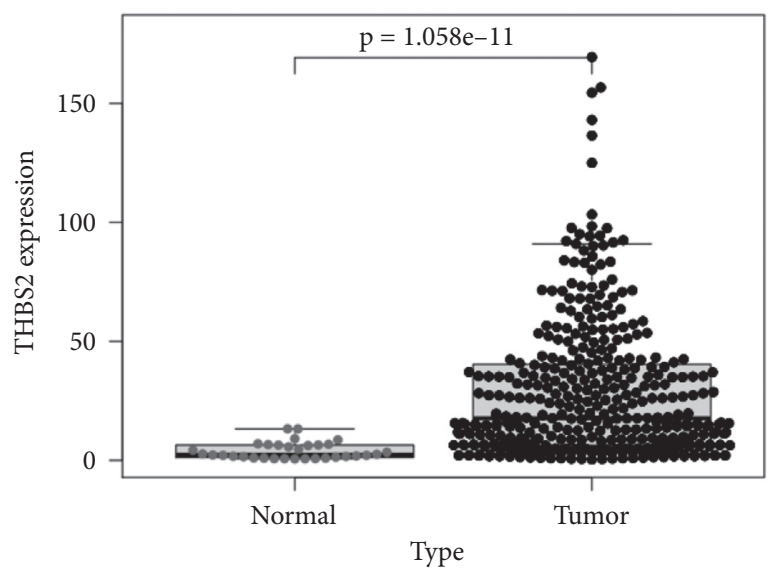

(a)

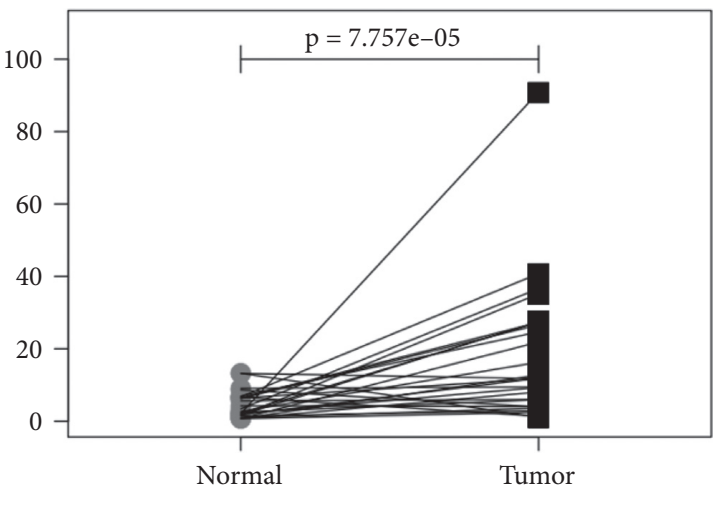

(b)

FIGURE 1: The level of THBS2 increased in gastric cancer tissues. (a) Data from TCGA website are deferential analyzed. (b) Data from TCGA website are paired deferential analyzed.

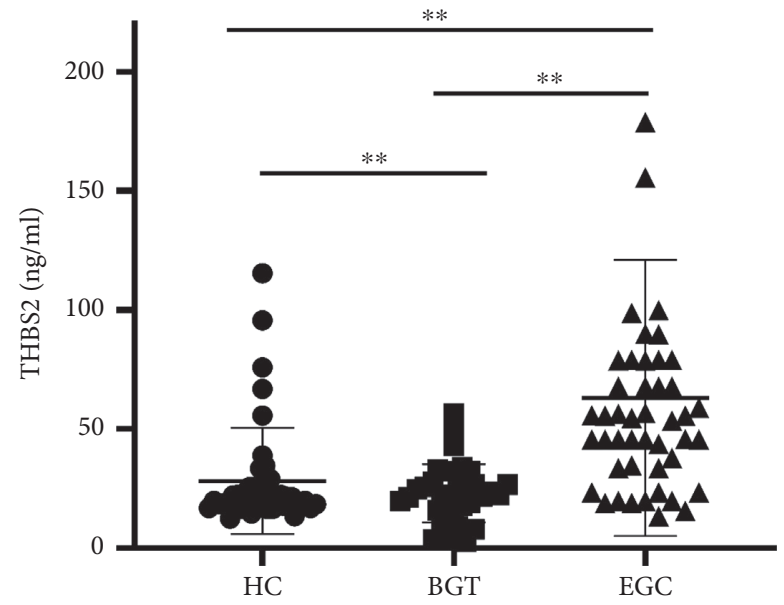

(a)

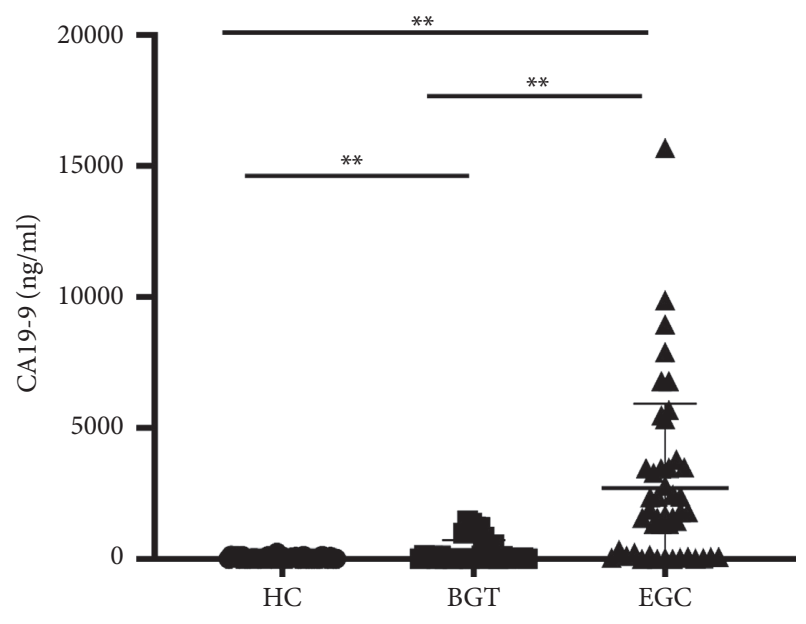

(b)

FIGURE 2: Increased level of THBS2 in serum of patients with early gastric cancer. (a) The level of serum THBS2 in various samples measured by the ELISA assay $(P<0.05)$. (b) The level of serum CA19-9 in various samples measured by the ELISA assay $(P<0.05)$.

\subsection{The Correlation between Serum THBS2 and CA19-9.} To reveal how serum THBS2 influences the detection of early gastric cancer, we made scatter plots based on the level of serum THBS2 in addition to CA19-9 and analyzed their relationship. Interestingly, there was no obvious correlation between THBS2 and CA19-9 in health controls sample $(P=0.157)$ as well as benign patients sample $(P=0.292)$, while there existed a significant correlation in patients with early gastric cancer $(P=0.04)$ (Figures $3(\mathrm{a})-3(\mathrm{c})$ ).

\subsection{The Detected Performance of THBS2 and CA19-9 for Early} Gastric Cancer. ROC curve analysis was employed to explore the performance of early diagnosis about serum THBS2 and CA19-9 in patients with GC (Figure 4(a)). Additionally, we listed all data obtained from the above analysis, including area under the curve (AUC) and asymptotic 95\% confidence interval in Table 2. Serum THBS2 possessed well capacity to forecast early gastric cancer with the value of AUC: 0.816 (95\% CI: 0.722-0.911). Serum CA19-9 distinguished patients with EGC from healthy control with the value of AUC of 0.901 (95\% CI: 0.833-0.968). Furthermore, serum THBS2 combined with CA19-9 as a marker could promote the performance of THBS2 or CA19-9 as an individual index (AUC: 0.951, 95\% CI: 0.912-0.989). Subsequently, we conducted the ROC curve to uncover whether THBS2 and CA19-9 differentiated patients with EGC from patients with BGT. As shown in Figure 4(b), the data showed that THBS2 or CA19-9 was able to predict early gastric cancer as an individual biomarker and combined THBS2 with CA19-9 could improve the diagnostic performance dramatically. All detailed data are given in Table 2.

3.5. THBS2 Plays a Vital Role in GC via Potentially Regulating MAPK and WNT Signaling Pathway. To further explore the functional mechanism of serum THBS2 on GC, we 


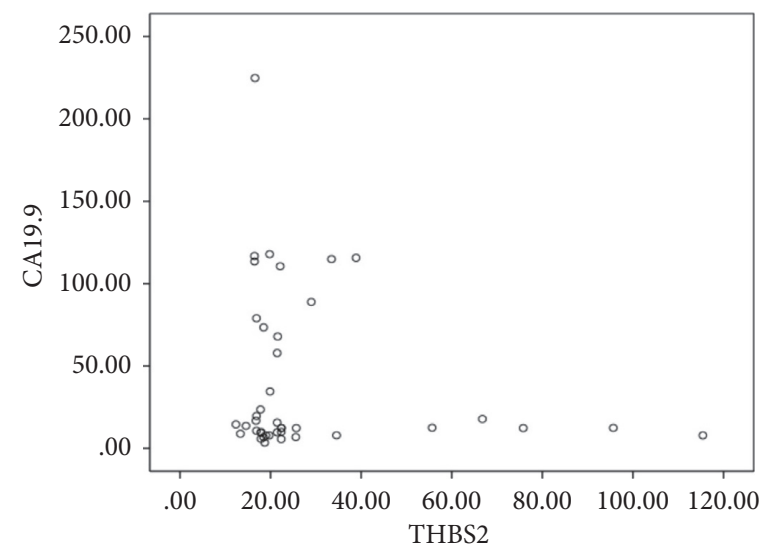

(a)

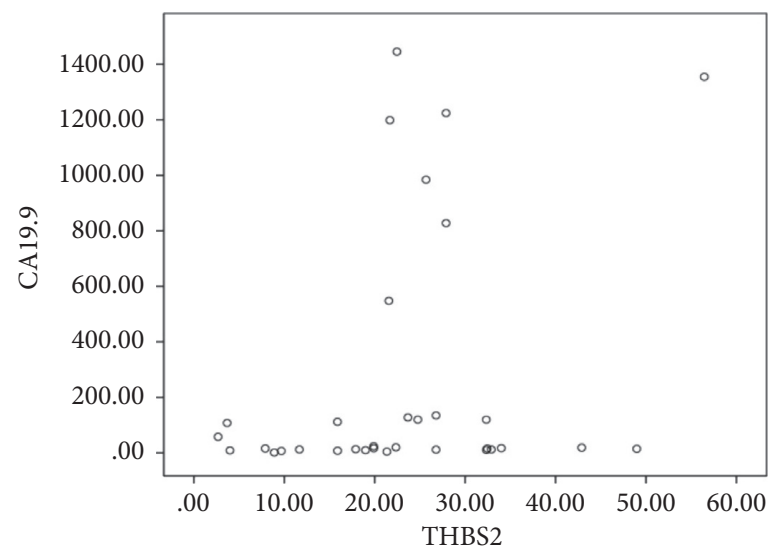

(b)

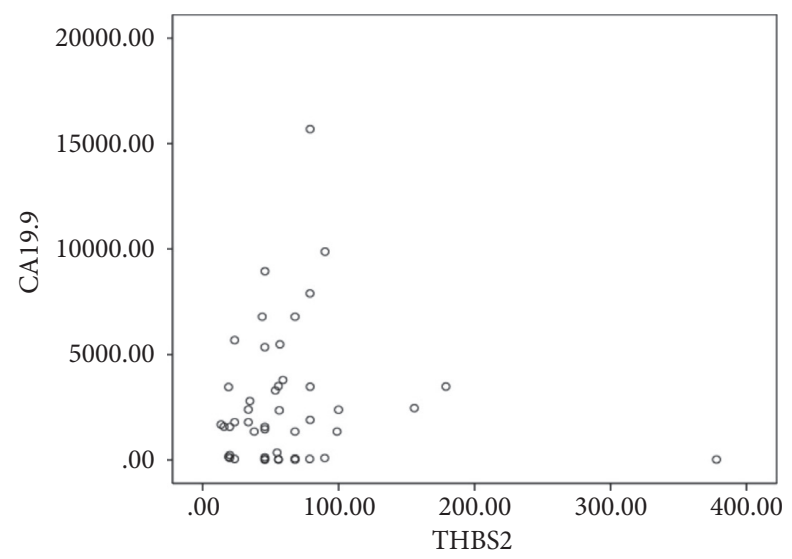

(c)

Figure 3: The correlation between serum THBS2 and CA19-9. (a) The correlation between THBS2 and CA19-9 in HC. (b) The correlation between THBS2 and CA19-9 in patients with BGT. (c) The correlation between THBS2 and CA19-9 in patients with EGC.

conducted the GSEA assay with data from the TCGA database and analyzed the THBS2-related pathway. The GSEA enrichment plot showed that high THBS2 expression was positively enriched in mitogen-activated protein kinase (MAPK) as well as Wnt- $\beta /$ catenin signaling pathway and exhibited a tightly positive relationship with multiple downstream genes involved in the pathway as mentioned earlier (Figures 5(a) and 5(b)).

\section{Discussion}

Increasing novel technologies, including liquid biopsy, have been employed to diagnose cancers; however, various proteins lack sensitivity in addition to specificity for applying into clinical practice [17]. More blood-based markers such as serum proteins possess the ability to offer information that is associated with the progression of cancers timely [18]. Therefore, serum biomarkers related to the early stage of cancers are required to diagnose and follow up patients with malignancy. Recently, emerging studies have revealed various markers that possess potential to detect gastric cancer in the early stage. For example, serum amyloid A cluster (SAA) and high mobility group box 1 (HMGB1) can be regarded as significant biomarkers for early diagnosis of GC [19]. Thymidine kinase 1 (TK1), CA19-9, and CA72-4 combined with other carcinoembryonic antigen exhibited better detection ability of GC and colorectal cancer (CRC) [20].

THBS2, one of the proteins belonging to thrombospondin family, has been reported to serve as a vital regulator of tumorigenesis [21]. In multiple cancers, decreasing the expression of THBS2 can trigger production of oncogenes or depress production of tumor suppresser genes. Upregulating THBS2 in cancer tissues is correlated to inhibit progression of tumors sometimes $[15,22,23]$. Simpson RE et al. found that THBS2 was a biomarker of pancreatic ductal adenocarcinoma and related to a high rate of dysplasia in sufferers with premalignant symptoms [24]. Liu et al. reported that it accelerates lung cancer progression through producing matrix metalloproteinase-13 [25]. In addition, serum THBS2 has been regarded as a valuable clinical marker for various cancers. For example, THBS2 has the ability to forecast prognosis for patients with colorectal cancer [26]. Downregulating THBS2 exerts utterly opposite functions on the clinical outcome of sufferers with gastric cancer [27, 28]. Nevertheless, no enough research studies devoted into uncovering the clinical value of serum THBS2 for diagnosing early gastric cancer. 


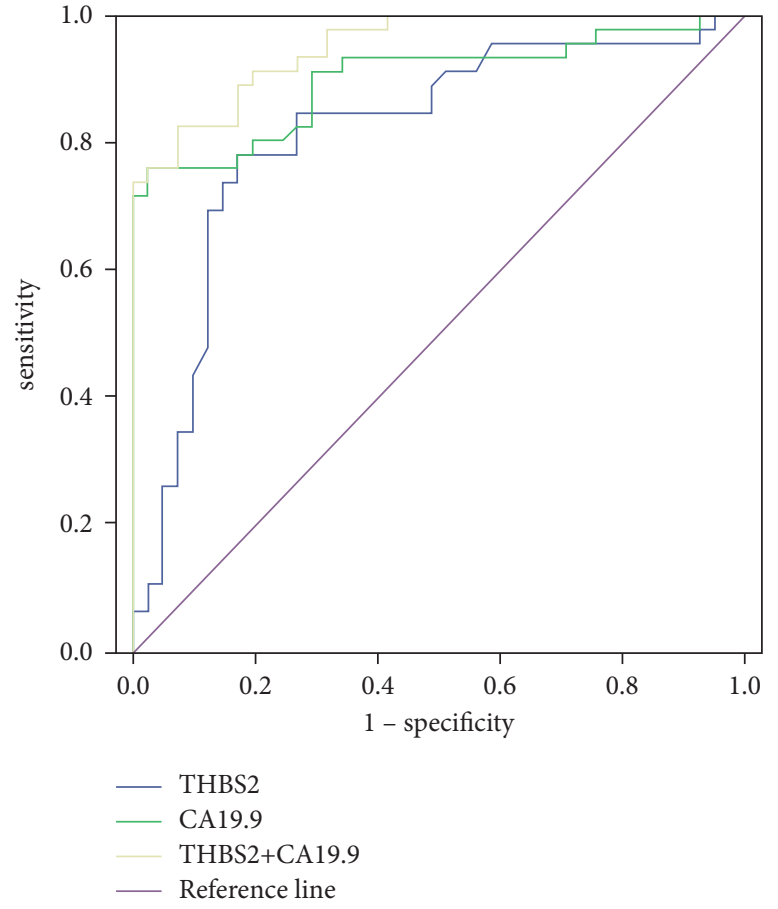

(a)

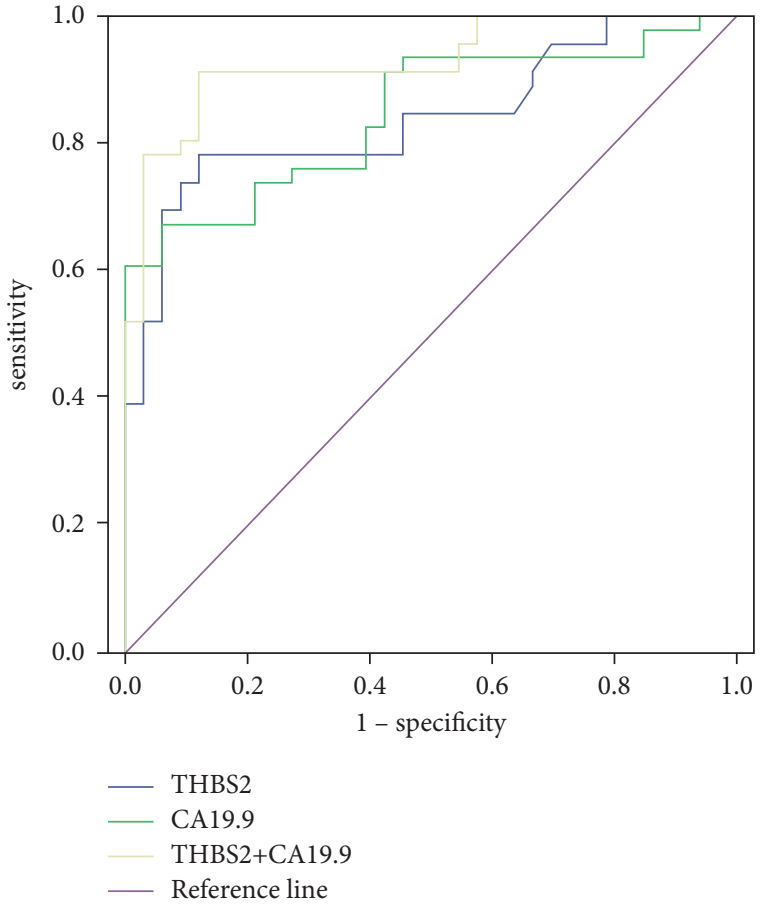

(b)

FIGURE 4: The detected performance of THBS2 and CA19-9 for early gastric cancer. (a) Diagnostic performance of THBST in addition to CA19-9 to differentiate early gastric cancer patients from a healthy control. (b) Diagnostic performance of THBST in addition to CA19-9 to differentiate early gastric cancer patients from benign gastric tumor patients.

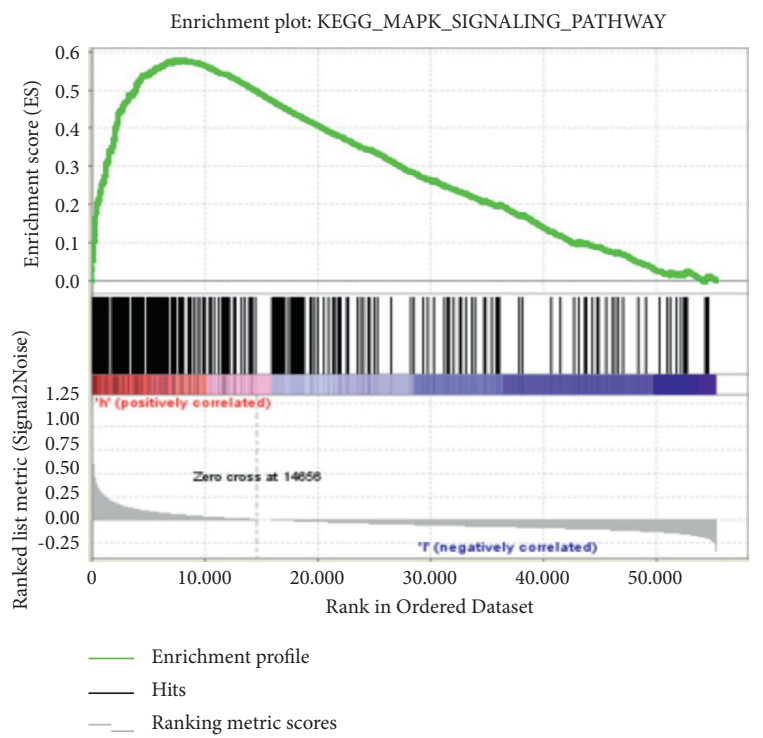

(a)

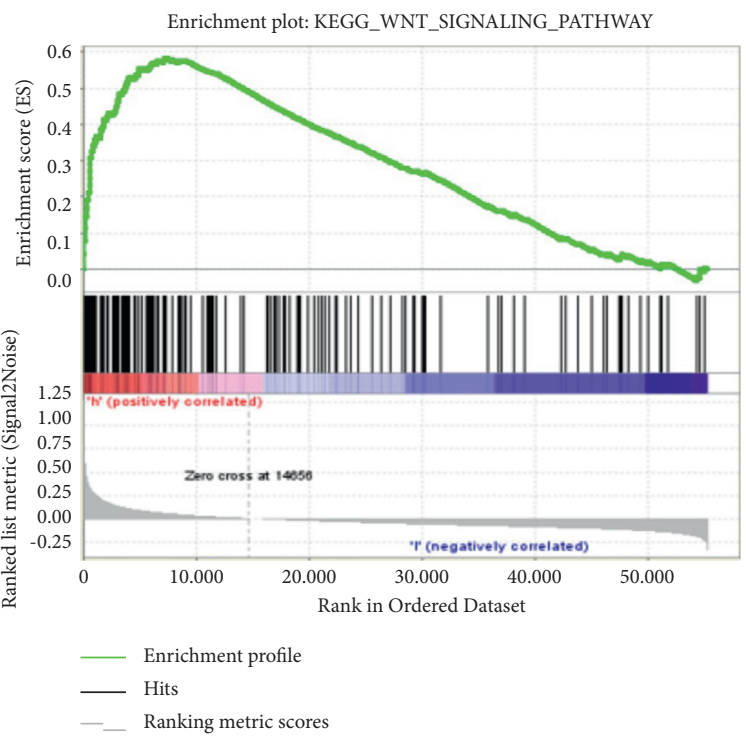

(b)

FIGURE 5: THBS2 playing a vital role in GC via potentially regulating MAPK and WNT signaling pathways. (a) Genes related to MAPK signaling pathway positively enriched by THBS2. (b) Genes related to WNT signaling pathway positively enriched by THBS2.

In our present study, we first analyzed data from the TCGA database and found that THBS2 was upregulated dramatically in gastric cancer tumor, which indicated that THBS2 might exert a crucial role in GC. To confirm the hypothesis, we collected tissue samples from 41 cases of $\mathrm{HC}$ and 33 cases of BGT in addition to 46 cases of EGC to measure the serum load of THBS2 by using ELISA assay. The results demonstrated that the level of serum THBST 


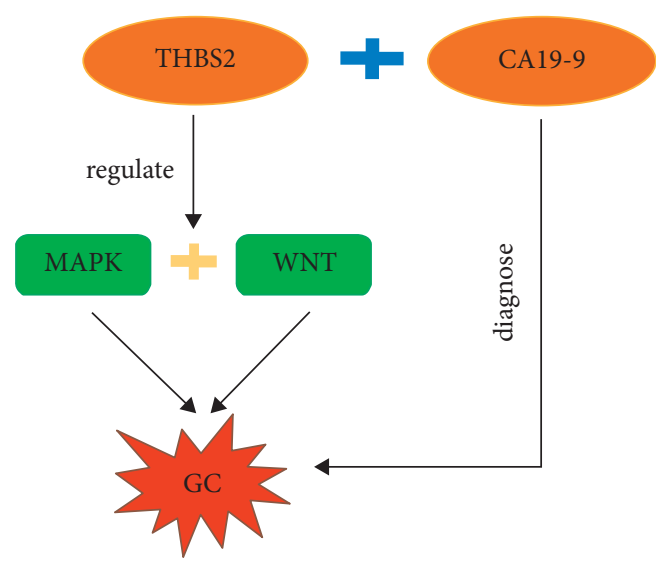

FIGURE 6: Schematic diagram of THBS2 regulating GC and THBS2 combined CA19-9 existing better performance in diagnosing GC.

was upregulated in patients with EGC compared to patients with BGT as well as HC significantly, implying that the increase of THBS2 was an important marker of GC. According to previous studies, the performance will get better to combine THBS2 and CA19-9 in detecting pancreatic ductal adenocarcinoma and distal cholangiocarcinoma [29]. Bamlet et al. discovered that THBS2 had good detection performance in tumors combined with CA19-9 [30]. Chen et al. revealed that THBS2 as one of biomarkers could be replenished by CA19-9 for diagnosing colorectal cancer [31]. Given these reporters, we assumed THBS2 combined CA19-9 may exert better performance in diagnosing GC. Subsequently, we further conducted the ELISA assay and found the load of CA19-9 in serum of patients diagnosed with EGC was higher than that in EGT as well as in $\mathrm{HC}$, which illustrated that cancer tissues were likely to produce more THBS2 in serum in accordance with former research studies $[32,33]$. In addition, there exhibited an obvious correlation between THBS2 and CA19-9 in patients with ECG $(P=0.04)$ but not in HC $(P=0.157)$ and patients with EGT $(P=0.292)$ according to correlation analysis, indicating that both THBS2 and CA19-9 play an essential role in diagnosis of early GC. Next, ROC curve analysis was employed to reveal that THBS2 possessed a comparable ability to differentiate patients with EGC from $\mathrm{HC}$ and patients with EGT to CA19-9. Additionally, the detective capacity was notably improved when we combined THBS2 and CA19-9 as the diagnostic marker. Moreover, GSEA was employed to uncover that THBS2 positively related with the MAPK and Wnt- $\beta /$ catenin signaling pathway, implying the potential regulatory mechanism of serum THBS2 on gastric cancer first, eventhough it was also necessary to conduct more experiments to explore the detailed mechanisms (Figure 6).

There are still some limitations to our study. For example, our investigation did not conduct sufficient experiments to explore the detailed functions of THBS2 combined CA19-9 for GC in vivo. Moreover, we have not identified the direct target gene for THBS2. Additionally, more attention need to be paid to identify that whether THBS2 combined with CA19-9 exert equally dramatic effects on early detection of other cancers.

\section{Conclusion}

In conclusion, our study uncovered that THBS2 was upregulated in GC tissues and positively regulated MAPK in addition to the WNT signaling pathway. Moreover, both THBS2 and CA19-9 could serve as significant and excellent biomarkers for detecting GC in the early stage. Additionally, THBS2 combined CA19-9 exhibited better performance in the diagnosis of early GC, which may improve diagnostic efficiency for patients with GC.

\section{Data Availability}

The data used to support the findings of this study are included within the article.

\section{Conflicts of Interest}

The authors declare that they have no conflicts of interest.

\section{Authors' Contributions}

Lanzhi Li and Jie Dong contributed equally to this work.

\section{References}

[1] L. A. Torre, F. Bray, R. L. Siegel, J. Ferlay, J. Lortet-Tieulent, and A. Jemal, "Global cancer statistics," CA: A Cancer Journal for Clinicians, vol. 65, pp. 87-108, 2012.

[2] W. Chen, R. Zheng, P. D. Baade et al., "Cancer statistics in China, 2015," CA: A Cancer Journal for Clinicians, vol. 66, no. 2, pp. 115-132, 2016.

[3] M. S. Sierra, P. Cueva, L. E. Bravo, and D. Forman, "Stomach cancer burden in central and South America," Cancer Epidemiology, vol. 44, no. Suppl 1, pp. S62-S73, 2016.

[4] F. Feng, L. Sun, G. Xu et al., "Is it reasonable to treat early gastric cancer with mucosal infiltration and well differentiation by endoscopic submucosal resection?" Journal of Gastrointestinal Surgery, vol. 19, no. 12, pp. 2111-2119, 2015.

[5] J. Wang, Y. Liu, W. Sun, Q. Zhang, T. Gu, and G. Li, "Plasma exosomes as novel biomarker for the early diagnosis of gastric cancer," Cancer Biomarkers, vol. 21, no. 4, pp. 805-812, 2018.

[6] L. Zhu, J. Qin, J. Wang, T. Guo, Z. Wang, and J. Yang, "Early gastric cancer: current advances of endoscopic diagnosis and treatment," Gastroenterology Research and Practice, vol. 2016, Article ID 9638041, 7 pages, 2016.

[7] X. Zhu, M. Lv, H. Wang, and W. Guan, "Identification of circulating microRNAs as novel potential biomarkers for gastric cancer detection: a systematic review and metaanalysis," Digestive Diseases and Sciences, vol. 59, no. 5, pp. 911-919, 2014.

[8] S.-B. Tian, J.-C. Yu, W.-M. Kang et al., "Combined detection of CEA, CA 19-9, CA 242 and CA 50 in the diagnosis and prognosis of resectable gastric cancer," Asian Pacific Journal of Cancer Prevention, vol. 15, no. 15, pp. 6295-6300, 2014.

[9] Z. Jin, W. Jiang, and L. Wang, "Biomarkers for gastric cancer: progression in early diagnosis and prognosis (review)," Oncology Letters, vol. 9, no. 4, pp. 1502-1508, 2015.

[10] W. K. Leung, M.-S. Wu, Y. Kakugawa et al., "Screening for gastric cancer in Asia: current evidence and practice," The Lancet Oncology, vol. 9, no. 3, pp. 279-287, 2008. 
[11] P. Bornstein, "Thrombospondins function as regulators of angiogenesis," Journal of Cell Communication and Signaling, vol. 3, no. 3-4, pp. 189-200, 2009.

[12] O. I. Stenina, E. J. Topol, and E. F. Plow, “Thrombospondins, their polymorphisms, and cardiovascular disease," Arteriosclerosis, Thrombosis, and Vascular Biology, vol. 27, no. 9, pp. 1886-1894, 2007.

[13] J. C. Adams and J. Lawler, "The thrombospondins," Cold Spring Harbor Perspectives in Biology, vol. 3, no. 10, Article ID a009712, 2011.

[14] J. Lawler and M. Detmar, "Tumor progression: the effects of thrombospondin-1 and -2," The International Journal of Biochemistry \& Cell Biology, vol. 36, no. 6, pp. 1038-1045, 2004.

[15] T. Tokunaga, M. Nakamura, Y. Oshika et al., "Thrombospondin 2 expression is correlated withinhibition of angiogenesis and metastasis of coloncancer," British Journal of Cancer, vol. 79, 1999.

[16] T. Chijiwa, Y. Abe, N. Ikoma et al., "Thrombospondin 2 inhibits metastasis of human malignant melanoma through microenvironment-modification in $\mathrm{NOD} / \mathrm{SCID} / \gamma$ Cnull (NOG) mice," International Journal of Oncology, vol. 34, 1992.

[17] E. Heitzer, S. Perakis, J. B. Geigl, and M. R. Speicher, "The potential of liquid biopsies for the early detection of cancer," Npj Precision Oncology, vol. 1, no. 1, p. 36, 2017.

[18] A. Li, T. Zhang, M. Zheng, Y. Liu, and Z. Chen, "Exosomal proteins as potential markers of tumor diagnosis," Journal of Hematology \& Oncology, vol. 10, no. 1, p. 175, 2017.

[19] A. A. Ghweil, H. A. Osman, M. H. Hassan et al., "Validity of serum amyloid A and HMGB1 as biomarkers for early diagnosis of gastric cancer," Cancer Management and Research, vol. 12, pp. 117-126, 2020.

[20] S. Ning, W. Wei, J. Li et al., "Clinical significance and diagnostic capacity of serum TK1, CEA, CA 19-9 and CA 72-4 levels in gastric and colorectal cancer patients," Journal of Cancer, vol. 9, no. 3, pp. 494-501, 2018.

[21] W. Naumnik, M. Ossolińska, I. Płońska, E. Chyczewska, and J. Nikliński, "Circulating thrombospondin-2 and FGF-2 in patients with advanced non-small cell lung cancer: correlation with survival," Advances in Experimental Medicine and Biology, vol. 833, pp. 9-14, 2015.

[22] G. Bocci, G. Francia, S. Man, J. Lawler, and R. S. Kerbel, "Thrombospondin 1, amediator of the antiangiogenic effects of low-dose metronomic chemotherapy," Proceedings of the National Academy of Sciences, vol. 100, 2003.

[23] M. Streit, L. Riccardi, P. Velasco et al., "Thrombospondin-2: a potent endogenous inhibitor o ftumor growth and angiogenesis," Proceedings of the National Academy of Sciences, vol. 96, no. 26, pp. 14888-14893, 1999.

[24] R. E. Simpson, M. T. Yip-Schneider, H. Wu et al., "Circulating Thrombospondin-2 enhances prediction of malignant intraductal papillary mucinous neoplasm," The American Journal of Surgery, vol. 217, no. 3, pp. 425-428, 2019.

[25] J.-F. Liu, C.-W. Lee, M.-H. Tsai et al., "Thrombospondin 2 promotes tumor metastasis by inducing matrix metalloproteinase-13 production in lung cancer cells," Biochemical Pharmacology, vol. 155, pp. 537-546, 2018.

[26] X. Wang, L. Zhang, H. Li, W. Sun, H. Zhang, and M. Lai, "THBS2 is a potential prognostic biomarker in colorectal cancer," Scientific Reports, vol. 6, no. 1, Article ID 33366, 2016.

[27] R. Sun, J. Wu, Y. Chen et al., "Down regulation of Thrombospondin2 predicts poor prognosis in patients with gastric cancer," Molecular Cancer, vol. 13, no. 1, p. 225, 2014.
[28] C. Zhuo, X. Li, H. Zhuang et al., "Elevated THBS2, COL1A2, and SPP1 expression levels as predictors of gastric cancer prognosis," Cellular Physiology and Biochemistry, vol. 40, no. 6, pp. 1316-1324, 2016.

[29] T. Y. S. Le Large, L. L. Meijer, R. Paleckyte et al., "Combined expression of plasma thrombospondin-2 and CA19-9 for diagnosis of pancreatic cancer and distal cholangiocarcinoma: a proteome approach," The Oncologist, vol. 25, pp. e634-e643, 2020.

[30] J. Kim, W. R. Bamlet, A. L. Oberg et al., "Detection of early pancreatic ductal adenocarcinoma with thrombospondin-2 and CA19-9 blood markers," Science Translational Medicine, vol. 9, no. 11, pp. 1-13, 2017.

[31] W. Fei, L. Chen, J. Chen et al., "RBP4 and THBS2 are serum biomarkers for diagnosis of colorectal cancer," Oncotarget, vol. 8, no. 54, pp. 92254-92264, 2017.

[32] T.-Y. Weng, C.-Y. Wang, Y.-H. Hung, W.-C. Chen, Y.-L. Chen, and M.-D. Lai, "Differential expression pattern of THBS1 and THBS2 in lung cancer: clinical outcome and a systematic-analysis of microarray databases," PLoS One, vol. 11, no. 8, Article ID e0161007, 2016.

[33] R. Navab, D. Strumpf, B. Bandarchi et al., "Prognostic geneexpression signature of carcinoma-associated fibroblasts in non-small cell lung cancer," Proceedings of the National Academy of Sciences, vol. 108, no. 17, pp. 7160-7165, 2011. 